\title{
Reliability and Inequality Measures for the Weimal Distribution
}

\author{
${ }^{1} \mathrm{~T}$. G. leren and ${ }^{2 *} \mathrm{~A}$. Yahaya \\ 1Department Of Statistics, Ahmadu Bello University Zaria, Nigeria \\ 2Department Of Statistics, Ahmadu Bello University Zaria, Nigeria \\ [Corresponding author: Email: abubakaryahaya@abu.edu.ng,ternagodfrey@gmail.com]
}

\section{ABSTRACT}

Reliability analysis basically deals with the probability of survival or failure (death). This article aimed at discussing both reliability and inequality measures from the Weimal distribution. The work has derived and discussed theoretically, expressions for the survival and hazard function of the Weimal distribution. The ordinary and incomplete moments of the distribution were also obtained. Lastly, some inequality measures for the distribution were derived using the moments of the distribution.

Keywords: Reliability; Inequality measures; Moments; survival function, hazard function, income distribution.

\section{INTRODUCTION}

In many applied sciences such as economics, engineering and finance, amongst others, modeling and analyzing lifetime data is crucial. Life time distributions are used to describe, statistically, the length of the life of a system. Reliability theory is based on the concept of understanding the reliability of systems and their individual components (Santiago, 2013). If inequality is assessed using a single inequality measure, a number of important dimensions of the change in inequality due to a certain policy will not be picked up (Gastwirth, 2016). Each inequality measure incorporates assumptions about the way in which income differences in different parts of the distribution are summarized. It is therefore desirable to calculate a wide range of inequality indexes incorporating different assumptions, but at the same time having a common theoretical foundation in order to thoroughly evaluate a redistribution policy (Gastwirth, 2016).
Since its introduction, the Lorenz curve (Lorenz, 1905) has been largely studied and has become a milestone of statistics. The methodological and applicative works on that subject are so numerous that it is very difficult to choose even a small selection. Aaberge (2000); Groves-Kirkby et al. (2009) and Jacobson et al. (2005) can be mentioned in order to highlight the importance of Lorenz curve regarding applications in different scientific fields. Nevertheless, several alternative tools for evaluating the inequality have been proposed in literature e.g, the inequality curve by Zenga (2007). The aim of this article is to derive reliability and inequality measures from the Weimal distribution.

\section{The Weimal Distribution}

According to leren and Yahaya (2017), the cumulative distribution function (cdf) and probability density function ( $p d f)$ of the Weimal distribution with scale parameter, $\alpha$, shape parameter, $\beta$, location parameter, $\mu \in \mathbb{R}$ and dispersion parameter $\sigma>0$ are given as:

$F(x)=F(x ; \alpha, \beta, \mu, \sigma)=1-\exp \left\{-\alpha\left[\frac{\Phi\left(\frac{x-\mu}{\sigma}\right)}{1-\Phi\left(\frac{x-\mu}{\sigma}\right)}\right]^{\beta}\right\}$

and

$f(x)=f(x ; \alpha, \beta, \mu, \sigma)=\frac{\alpha \beta}{\sigma} \phi\left(\frac{x-\mu}{\sigma}\right) \frac{\Phi\left(\frac{x-\mu}{\sigma}\right)^{\beta-1}}{\left(1-\Phi\left(\frac{x-\mu}{\sigma}\right)\right)^{\beta+1}} \exp \left\{-\alpha\left[\frac{\Phi\left(\frac{x-\mu}{\sigma}\right)}{1-\Phi\left(\frac{x-\mu}{\sigma}\right)}\right]^{\beta}\right\}$

respectively. 


\section{leren and Yahaya: Reliability and Inequality Measures for the Weimal Distribution}

Equations (1) and (2) can be written as a mixture of exponentiated density functions (Exponentiated Normal, EN) as represented by equation (3) and (4) below:

$\mathrm{F}(\mathrm{x})=\sum_{r=0}^{\infty} \eta_{r} \Phi\left(\frac{x-\mu}{\sigma}\right)^{r}=\sum_{r=0}^{\infty} \eta_{r} H(x ; r, \mu, \sigma)$

Where $\eta_{r}=\sum_{j, k=0}^{\infty} w_{j, k} s_{r(\beta(k+1)+j)}$

By differentiating equation (3) and changing indices, we can obtain the $p d f$ of the Weimal distribution as:

$f(x)=\frac{d F(x)}{d x}=\sum_{r=0}^{\infty} \eta_{r} r \phi\left(\frac{x-\mu}{\sigma}\right) \Phi\left(\frac{x-\mu}{\sigma}\right)^{r-1}=\sum_{r=0}^{\infty} \eta_{r} h(x ; r, \mu, \sigma)$

Where $\eta_{r}=\sum_{j, k=0}^{\infty} w_{j, k} s_{r(\beta(k+1)+j)}$ and $\sum_{r=0}^{\infty} \eta_{r}=1$

Equation (3) and (4) are the cdf and pdf of the Weimal distribution defined as a linear combination of $E N$ pdfs. So, several properties of the Weimal distribution can be obtained by following those properties of $E N$ distribution.

\section{RELIABILITY ANALYSIS OF THE WEIMAL DISTRIBUTION}

The survival function

The survival function, also known as the reliability function in engineering, is the characteristic of an explanatory variable that maps a set of events, usually associated with mortality or failure of some system onto time. It is the probability that the system will survive beyond a specified time. This function is defined as follows:

$S(x)=1-F(x)$ where $F(x)$ is the $c d f$ of the required distribution. Using the cdf of the Weimal distribution above, the survival function of a Weimal distribution is given by:

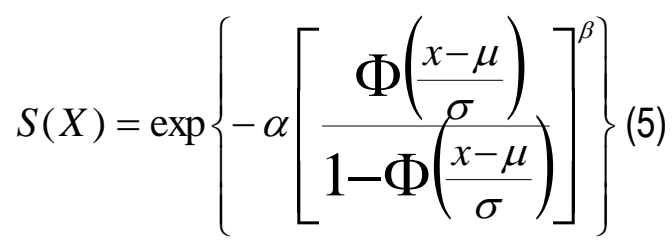

A plot for the survival function of the Weimal distribution at different parameter values is as shown in Figure 1. From Figure 1, we can see that the value of the survival function equals one (1) at initial time or early age and it decreases as $X$ increases and equals zero (0) as $X$ becomes larger.

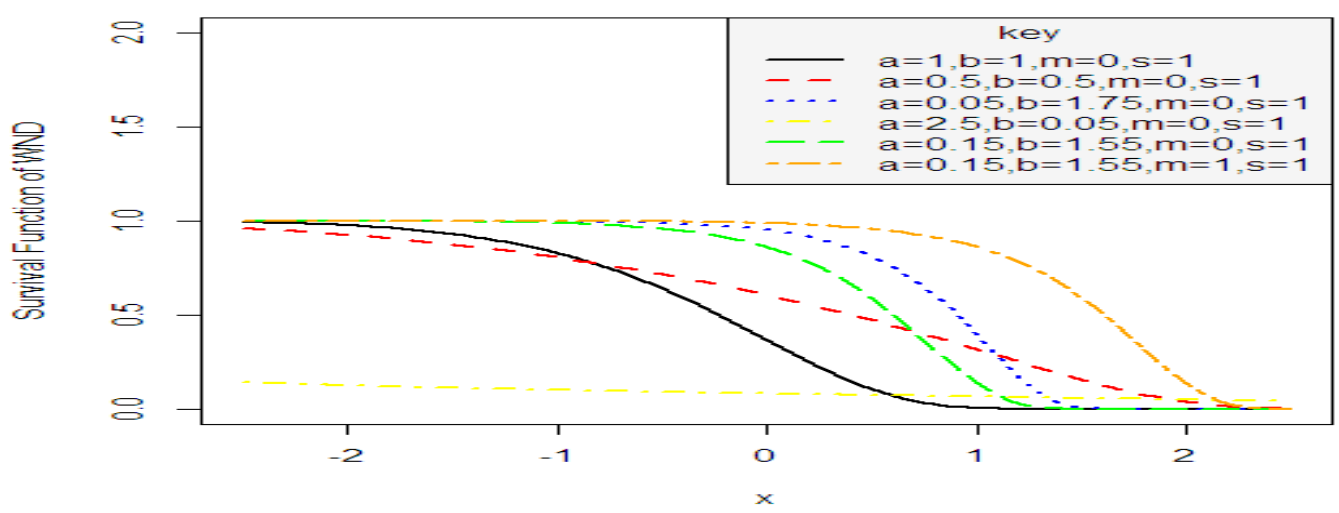

Figure 1: Survival function of the Weimal distributionat different parameter values where $a=\alpha, b=\beta, m=\mu$ and $s=\sigma$. 
The Hazard Function

The hazard function is defined as the probability per unit time that a case which has survived to the beginning of the respective interval will fail in that interval. Specifically, it is computed as the number of failures per unit time in the respective interval, divided by the average number of surviving cases at the mid-point of the interval.

Mathematically, the hazard function for a random variable $X$ is defined as:

$$
h(x)=\frac{f(x)}{S(x)}=\frac{f(x)}{1-F(x)}
$$

Hence the hazard function associated with the Weimal distributionfrom Equation (1) and Equation(2) is

$$
h(x)=\frac{\alpha \beta}{\sigma} \phi\left(\frac{x-\mu}{\sigma}\right)\left[\Phi\left(\frac{x-\mu}{\sigma}\right)\right]^{\beta-1}
$$

The following are some possible curves for the hazard rate at various values of the model parameters

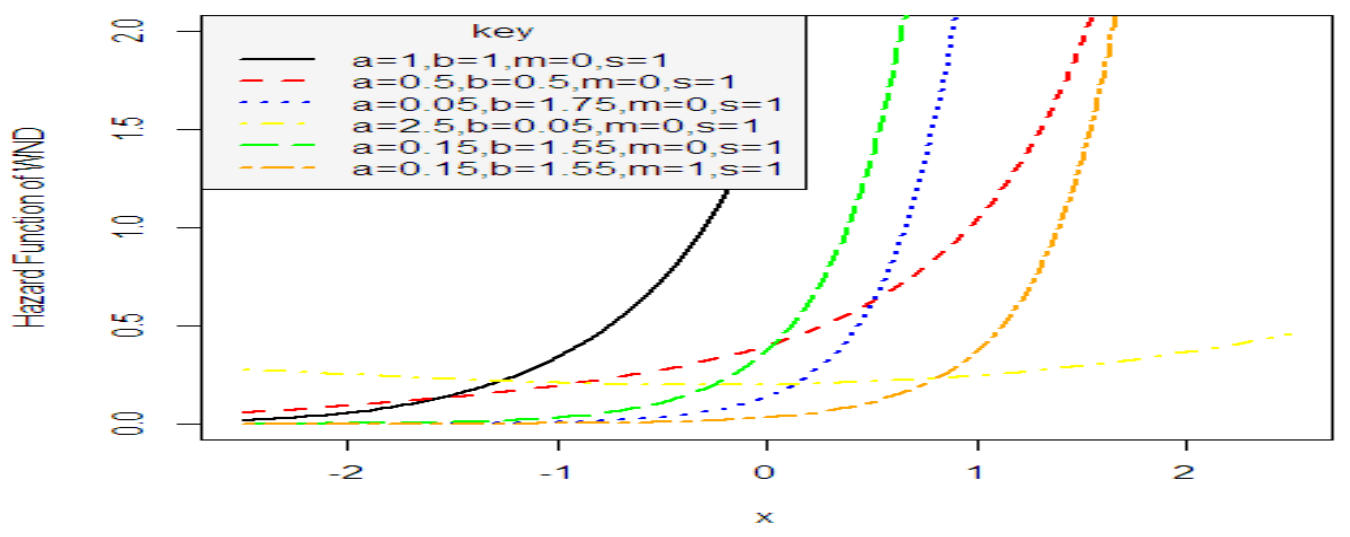

Figure 2: Hazard function of theWeimal distributionat different parameter values where $a=\alpha, b=\beta, m=\mu$ and $s=\sigma$.

From Figure 2, we can see that the value of the hazard function increases when $X$ increases. It gets higher as the value of $X$ increases. This means that the Weimal distribution would be appropriate in modeling time or age-dependent events, where risk or hazard increases with time or age. Many examples are found in products that fail as a result of the age of those components.

\section{MOMENTS AND INEQUALITY MEASURES}

In this section, we present moments and inequality measures for the Weimal distribution.

\section{MOMENTS}

Ordinary or non-central Moments are used to study some of the most important features and characteristics of a random variable such as mean (central tendency measure), variance (dispersion measure), skewness (Sk) and kurtosis $(\mathrm{Ku})$.

Let $X_{1}, X_{2}, \ldots, X_{n}$ denote a random sample from the standard Weimal distribution which is obtained from equation (2) for $\mu=0$ and $\sigma=1$, that is $X \sim W N(\alpha, \beta, 0,1)$.

The $n^{\text {th }}$ raw or non-central moments of $X$ can be obtained as: 


\section{Ieren and Yahaya: Reliability and Inequality Measures for the Weimal Distribution}

$=E\left[X^{n}\right]=\sum_{r=0}^{\infty} r \eta_{r} \int_{-\infty}^{\infty} X^{n} \phi(x) \Phi(x)^{r-1} d x$

Now, substituting for $\phi(x)$ and $\Phi(x)$ in equation (8), using binomial expansionand simplifying, we have:

$\mu_{n}^{\prime}=E\left[X^{n}\right]=\sum_{r=0}^{\infty} \sum_{p=0}^{r-1} r \eta_{r} \frac{2^{-[r-1]}}{\sqrt{2 \pi}}\left(\begin{array}{c}r-1 \\ p\end{array}\right) I(n, p)$

Where $I(n, p)$ represents the $(n, p)^{\text {th }}$ probability weighted moment $(P W M)$ for any $n$ and $p$ positive

integers of the standard normal distribution and is found as follows:

$I(n, p)=\pi^{-\frac{p}{2}} 2^{\left(\frac{n-1}{2}\right)+p} \sum_{m_{1}=0}^{\infty} \ldots \sum_{m_{p}}^{\infty} \frac{(-1)^{m_{1}+\cdots+m_{p}}}{\left(2 m_{1}+1\right) \ldots\left(2 m_{p}+1\right) m_{1} ! \ldots m_{p} !} \Gamma\left(m_{1}+\cdots+m_{p}+\frac{p+n+1}{2}\right)$

Now, according to Nadarajah (2008),

$(f)_{n}=\Gamma(f+n) /(f)$

and

$F_{(A)}^{(n)}\left(a ; b_{1}, \ldots, b_{n} ; c_{1}, \ldots, c_{n} ; x_{1}, \ldots, x_{n}\right)=\sum_{m_{1}=0}^{\infty} \ldots \sum_{m_{n}}^{\infty} \frac{a_{m 1+\ldots+m_{n}}(b 1) m_{1} \ldots . .(b n) m_{n}}{\left(c_{1}\right) m_{1} \ldots \ldots .\left(m_{n}\right) m_{n}} \frac{x_{1}^{m 1} \ldots \ldots x_{n}^{m n}}{m_{1} ! \ldots \ldots m_{n} !}$

where (12) is the Lauricella function of type A (Exton, 1978).

Using these definitions in (11) and (12) we can express (10) as:

$I(n, p)=\pi^{-\frac{p}{2}} 2^{\left(\frac{n-1}{2}\right)+p} F_{(A)}^{(p)}\left(\frac{p+n+1}{2} ; \frac{1}{2}, \ldots, \frac{1}{2} ; \frac{3}{2}, \ldots, \frac{3}{2} ;-1, \ldots,-1\right)$

for $p+n$ is even.

Combining (9) and (13), we can express the $n^{\text {th }}$

1978) demonstrated by Nadarajah (2008),

moment of the standard Weimal distribution in Nadarajah and Kotz (2006), Pescim et al. (2015), terms of the Lauricella function of type $A$ (Exton,

$\mu_{n}^{\prime}=E\left[X^{n}\right]=$

$\sum_{r=0}^{\infty} \sum_{p=0, p+n, \text { even }}^{r-1}\left(\begin{array}{c}r-1 \\ p\end{array}\right) r \eta_{r} \pi^{-\frac{(p+1)}{2}} 2^{\frac{n}{2}-r+p} \Gamma\left(\frac{p+n+1}{2}\right) F_{(A)}^{(p)}\left(\frac{p+n+1}{2} ; \frac{1}{2}, \ldots, \frac{1}{2} ; \frac{3}{2}, \ldots, \frac{3}{2} ;-1, \ldots,-1\right)$

Incomplete moments: We also derive an expression for the $n^{\text {th }}$ incomplete moment of $X$ for the Weimal distribution as given below:

$$
\begin{gathered}
E\left[X^{n} \mid X \leq x\right]=I_{X}(y)=\int_{0}^{y} x^{n} f(x) d x \\
E\left[X^{n} \mid X \leq x\right]=\sum_{j, k=0}^{\infty} r \eta_{r} \int_{0}^{y} x^{n} \phi(x) \Phi(x)^{r-1} d x
\end{gathered}
$$

Note that we can write $\Phi(x)$ using power series as:

$\Phi(x)=\sum_{j=0}^{\infty} a_{j} x^{j}$

where $a_{0}=\left(1+\sqrt{\frac{2}{\pi}}\right)^{-1} / 2, a_{2 j+1}=(-1)^{j} /\left[\sqrt{2 \pi} 2^{j}(2 j+1) j !\right]$, for $\mathrm{j}=0,1,2, \ldots$ and $a_{2 j}=0$ for $j=1,2, \ldots \ldots$ 


\section{Nigerian Journal of Basic and Applied Science (December, 2016), 24(2): 53-59}

Using the identity given by Gradshteyn and Ryzhik (2007) for power series raised to a positive integer i, we have:

$\left(\sum_{j=0}^{\infty} C_{j} x^{j}\right)^{i}=\sum_{j=0}^{\infty} C_{i, j} x^{j}$

Where the coefficient $C_{i, j}$ (for $\mathrm{i}=1,2, \ldots$ ) are easily obtained from the recurrence equation as:

$C_{i, j}=\left(j a_{0}\right)^{-1} \sum_{m=0}^{j}[m(i+1)-j] a_{m} C_{i, j-m}$

and $C_{i, 0}=a_{0}^{i}$.

Hence, using the expressions in (16) and (17), it implies that:

$\Phi(x)^{r-1}=\sum_{j=0}^{\infty} C_{r-1, j} x^{j}$

where the coefficients $C_{r-1, j}$ can be determined from the recurrence equation (18). Thus using (19) and simplifying the integral, it follows from

(15) that the $n^{\text {th }}$ incomplete moment of $X$ can be obtained as:

$E\left[X^{n} \mid X \leq x\right]=I_{X}(y)=\sum_{j, r=0}^{\infty} r \eta_{r} C_{r-1, j} \int_{0}^{y} x^{j+n} \exp \left(-\frac{x^{2}}{2}\right) d x$

Elementary integration and algebra shows that:

$E\left[X^{n} \mid X \leq x\right]=I_{X}(y)=\frac{1}{\sqrt{2 \pi}} \sum_{j, r=0}^{\infty} r \eta_{r} C_{r-1, j} 2^{\frac{1}{2}(j+n-1)} \Gamma\left(\frac{j+n+1}{2}, \frac{y^{2}}{2}\right)$

$E\left[X^{n} \mid X \leq x\right]=I_{X}(y)=\pi^{-\frac{1}{2}} \sum_{j, r=0}^{\infty} r \eta_{r} 2^{\frac{1}{2}(j+n)-1} \Gamma\left(\frac{j+n+1}{2}, \frac{y^{2}}{2}\right)$

Where $\Gamma(s, x)=\int_{x}^{\infty} t^{s-1} e^{-t} d t$ denotes the complementary incomplete gamma function.

INEQUALITY MEASURES

Lorenz and Bonferroni curves are income inequality measures that are widely useful and applicable to some other areas including reliability, demography, medicineand insurance (see, Bonferroni, 1930). Also Zenga curve

Lorenz curve, $L F(x)=\frac{\int_{0}^{x} t f(t) d t}{E[X]}=\frac{E_{X \leq x}[X]}{E[X]}$

Bonferroni curve, $B(F(x))=\frac{\int_{0}^{x} t f(t) d t}{F(x) E[X]}=\frac{E_{X \leq x}[X]}{F(x) E[X]}=\frac{L F(x)}{F(x)}$

and introduced by Zenga (2007) is another widely used inequality measure. In this section, we have derived the Lorenz, Bonferroni and Zenga curves for the Weimal distribution. The Lorenz, Bonferroni and Zenga curves are defined, respectively, by the following expressions: 
Zenga curve, $A(x)=1-\frac{\mu^{-}(x)}{\mu^{+}(x)}$

Where

$\mu^{-}(x)=\frac{\int_{0}^{x} t f(t) d t}{F(x) E[X]}=\frac{E_{X \leq x}[X]}{F(x)}$ and $\mu^{+}(x)=\frac{\int_{x}^{\infty} t f(t) d t}{1-F(x)}=\frac{E[X]-E_{X>x}[X]}{1-F(x)}$ are the lower and upper means. Using these results for the Weimal distribution, we obtain the defined curves as follows:

Lorenz curve for Weimal distribution is obtained as;

$$
L F(x)=\frac{\pi^{-\frac{1}{2}} \sum_{j, r=0}^{\infty} r \eta_{r} 2^{\frac{1}{2}(j+n)-1} \Gamma\left(\frac{j+n+1}{2}, \frac{y^{2}}{2}\right)}{\sum_{r=0}^{\infty} \sum_{p=0}^{r-1} r \eta_{r}\left(\begin{array}{c}
r-1 \\
p
\end{array}\right) \pi^{-\frac{(p+1)}{2}} 2^{\frac{1}{2}-r+p} \Gamma\left(\frac{p+2}{2}\right) F_{(A)}^{(p)}\left(\frac{p+2}{2} ; \frac{1}{2}, \ldots, \frac{1}{2} ; \frac{3}{2}, \ldots, \frac{3}{2} ;-1, \ldots,-1\right)}
$$

Bonferroni curve for Weimal distribution is obtained as:

$$
B F(x)=\frac{\pi^{-\frac{1}{2}} \sum_{j, r=0}^{\infty} r \eta_{r} 2^{\frac{1}{2}(j+n)-1} \Gamma\left(\frac{j+n+1}{2}, \frac{y^{2}}{2}\right)}{\left(\sum_{r=0}^{\infty} \eta_{r} \Phi(x)^{r}\right) \sum_{r=0}^{\infty} \sum_{p=0}^{r-1} r \eta_{r}\left(\begin{array}{c}
r-1 \\
p
\end{array}\right) \pi^{-\frac{(p+1)}{2}} 2^{\frac{1}{2}-r+p} \Gamma\left(\frac{p+2}{2}\right) F_{(A)}^{(p)}\left(\frac{p+2}{2} ; \frac{1}{2}, \ldots, \frac{1}{2} ; \frac{3}{2}, \ldots, \frac{3}{2} ;-1, . .,-1\right)}
$$

Zenga curve for the Weimal distribution is obtained as:

$$
\begin{aligned}
& A(x)=1-\left[\frac{\frac{E[X \mid X<x]}{F(x)}}{\frac{E[X]-E X \mid X \leq x]}{1-F(x)}}=1-\frac{(1-F(x))(E[X \mid X \leq x])}{F(x)(E[X]-E[X \mid X \leq x])}\right] \\
& \text { Where } E[X \mid X \leq x]=\pi^{-\frac{1}{2}} \sum_{j, r=0}^{\infty} r \eta_{r} 2^{\frac{1}{2}(j+n)-1} \Gamma\left(\frac{j+n+1}{2}, \frac{y^{2}}{2}\right) \\
& E[X]=\sum_{r=0}^{\infty} \sum_{p=0}^{r-1} r \eta_{r}\left(\begin{array}{c}
r-1 \\
p
\end{array}\right) \pi^{-\frac{(p+1)}{2}} 2^{\frac{1}{2}-r+p} \Gamma\left(\frac{p+2}{2}\right) F_{(A)}^{(p)}\left(\frac{p+2}{2} ; \frac{1}{2}, . ., \frac{1}{2} ; \frac{3}{2}, \ldots, \frac{3}{2} ;-1, . .,-1\right), \text { and } \\
& F(x)=\sum_{r=0}^{\infty} \eta_{r} \Phi(x)^{r} \text { for } \eta_{r}=\sum_{j, k=0}^{\infty} w_{j, k} S_{r}(\beta(k+1)+j)
\end{aligned}
$$

\section{CONCLUSION}

In this paper, we propose reliability and inequality measures widely used in Biology, engineering and economics respectively from the newly proposed Weimal distribution. This study has been done theoretically for both reliability and inequality measures. We have successfully derived explicit functions or expressions for the survival and hazard function of the Weimal distribution which are intensively useful in reliability theory. We further developed graphical descriptions of these functions to showcase their practical applications and we discovered after studying the plots for the survival and hazard functions that the Weimal distribution would be appropriate in modeling or analyzing time or agedependent events (random variables), where probability of survival decreases as time 


\section{Nigerian Journal of Basic and Applied Science (December, 2016), 24(2): 53-59}

increases while that of hazard or failure rate increases as time or age grows or increases. We have also presented the $n^{\text {th }}$ raw or general moments and the $n^{\text {th }}$ incomplete moments of the Weimal density function which are useful for studying the shape characteristic of distributions of random variables and also for calculation of some inequality measures. We also used the derived moments to obtain mathematically some popular inequality measures for the Weimal distribution including; Lorenz, Bonferroni and Zenga curves which are generally used for income distribution in the field of Economics.

\section{REFERENCES}

Aaberge, R. (2000). Characterizations of Lorenz curves and income distributions. Social Choice and Welfare, 17: 639-653

Bonferroni, E. (1930). Elementi di statistica generale. Libreria Seber,Firenze.

Bourguignon, M., R.B. Silva and G.M., Cordeiro. (2014). The Weibull-G Family of Probability Distributions. Journal of Data Science, 12: 53-68.

Exton, H. (1978). Handbook of Hypergeometric integrals: Theory, Applications, Tables, Computer programs. New York: Halsted press, p.200.

Gastwirth, J. L. (2016). Measures of Economic Inequality Focusing on the Status of the Lower and Middle Income Groups. Statistics and Public Policy, DOI:10.1080/2330443X.2016.1213148

Gradshteyn, I. S. and Ryzhik, I. M. (2007): Table of integrals, series and products. Sandiego: academic press, p.315.

Groves-Kirkby, C., Denman, A., and Phillips, P. (2009). Lorenz curve and Gini coefficient: Novel tools for analysing seasonal variation of environmental radon gas. Journal of Environmental Management, 90: 2480-2487

leren, T. G. and Yahaya, A. (2017). The Weimal Distribution: its properties and applications. Journal of the Nigeria Association of Mathematical Physics, 39: 135-148.

Jacobson, A., Milman, A., and Kammen, D. (2005). Letting the (energy) Gini out of the bottle:
Lorenz curves of cumulative electricity consumption and Gini coefficients as metrics of energy distribution and equity. Energy Policy, 33: 1825-1832

Lima, M. S., Cordeiro, G. M and Ortega, E. M. M (2015): A New Extension of The Normal Distribution. Journal of Data Science, 13, 385-408.

Lorenz, M. (1905). Methods of measuring the concentration of wealth. American StatisticalAssociation, 9: 209-219

Nadarajah, S. (2005): A Generalized Normal Distribution. Journal of Applied Statistics, 32, 685-694.

Nadarajah, S. (2008): Explicit expressions for moments of order statistics. Statistics and Probability letters,Amsterdam, 78: 195205.

Nadarajah, S. and Kotz, S. (2006): The exponentiated type distribution. Acta Applicandae Mathematicae, Amsterdam, 92: 97-111.

Pescim, R.R. and Nadarajah, S. (2015). The Kummer Beta Normal: A New Skew Model. Journal of Data Science, 13: 509532.

Santiago, R. (2013). Reliability theory: Properties of probability distributions for lifetimes of systems of components. Thesis, Rochester Institute of Technology. Accessed from http://scholarworks. rit.edu/theses

Zenga, M. (2007). Inequality curve and inequality index based on the ratios between lower and upper arithmetic means. Statistica and Applicazioni, 5: 3-27. 